\title{
Surgical outcome and clinical profile of emergency versus elective cases of colorectal cancer in College of Medical Sciences, Nepal
}

\author{
Kumar S, ${ }^{1}$ Kafle $\mathrm{P}_{,}{ }^{2}$ Patowary BN, ${ }^{3}$ Belbase N,, Agrawal S, ${ }^{2}$ Shrestha $S,{ }^{2}$ Maharjan $\mathbf{N}^{2}$ \\ ${ }^{1}$ Assistant Professor, ${ }^{2}$ Residents, ${ }^{3}$ Professor and Head, ${ }^{4}$ Lecturer, Department of Surgery, College of \\ Medical Sciences, Bharatpur, Chitwan Nepal.
}

\section{ABSTRACT \\ Background}

Patients who undergo emergency colorectal cancer surgery has poor outcome compared to elective surgery, both in terms of morbidity and mortality. Approximately 15 to $30 \%$ of colorectal cancers present as an emergency, most often as obstruction or perforation.

\section{Objective}

To compare surgical outcome and clinical profiles of emergency and elective cases for colorectal cancer.

\section{Methods}

Retrospective analysis of 34 cases who underwent surgery for colorectal cancer between December 2011 to January 2013was carried out and their surgical outcomes, clinical presentation, demographic profile were analyzed.

\section{Results}

The total numbers of patients included in this study were 34 . Out of which $52.94 \%(n=18)$ were emergency cases and $47.05 \%(n=16)$ were elective. Male female ratio was 3:1 in emergency cases and $2.6: 1$ in elective cases. Per rectal bleeding (56\%) and altered bowel habit (31.25\%) was predominant clinical presentation in elective cases whereas intestinal obstruction (55.55\%) and peritonitis (22.22\%) were predominant clinical presentation in emergency cases. In emergency cases most of the tumors were located in left side $(77.77 \%)$ and in elective cases rectum was common site (37.5\%). Left hemicolectomy was the commonest surgery performed (72.22\%) in emergency set up. In elective cases, right hemicolectomy, left hemicolectomy, APR and LAR was done in $31.25 \%, 31.25 \%, 25 \%$ and $25 \%$ cases respectively. In the emergency group $11.11 \%(n=2)$ developed enterocutaneous fistula and early mortality within 30 days was observed in $5 \%(n=1)$ of emergency cases only.

\section{Conclusion}

In emergency conditions, colorectal cancer presented with intestinal obstruction where as elective cases presented with per rectal bleeding and altered bowel habits. Compared with the elective patients, the emergency patients had higher rate of morbidity and mortality. Because of higher incidence of colorectal cancer in our institution, in all emergency cases who presents with features of intestinal obstruction and peritonitis, we have to rule out colorectal cancer especially if the patients are elderly. Therefore, we should encourage screening programme for early detection of colorectal cancers for better outcome of surgery.

Keywords: Colorectal cancer, Surgical outcome, emergency versus elective, mortality and morbidity.

Correspondence to: Dr.Sujit Kumar

E-mail: drsujit1755@gmail.com 
Journal of College of Medical Sciences-Nepal, 2013, Vol-9, No-2,

\section{INTRODUCTION}

Colorectal cancer remains a huge diagnostic and therapeutic issue worldwide. World epidemiological data indicates a constant increase in morbidity in recent decades. ${ }^{1}$ Colorectal cancer is the most common malignancy in the gastrointestinal tract. In the United States, colorectal cancer ranks third in terms of both gender-specific annual cancer incidence and cancer mortality. ${ }^{2}$ Worldwide, colorectal cancer shows large geographic differences, with a crude incidence of 6.5/7.7 cases per 100,000 females/males in less developed areas as opposed to 50.9/60.8 in more developed regions. The lifetime risk for developing colorectal cancer in the United States is 1 in 17 for men and 1 in 19 for women. The risk for developing invasive colorectal cancer increases with age, with more than $90 \%$ of new cases being diagnosed in patients older than 50 years.

The signs and symptoms of colon cancer are varied, nonspecific, and somewhat dependent on the location of the tumor in the colon as well as the extent of constriction of the lumen caused by the cancer. During the past several decades, the incidence of cancer in the right colon has increased in comparison to cancer arising in the left colon and rectum. This is an important consideration, in that at least half of all colon cancers are located proximal to the area that can be visualized by the flexible sigmoidoscope. Bleeding from right-sided colon tumors can cause dark, tarry stools. Often, the bleeding is asymptomatic and detected only by anemia discovered by a routine hemoglobin determination. ${ }^{2}$ Cancers located in the left colon are often constrictive in nature. Patients with leftsided colon cancers may notice a change in bowel habit, rectal bleeding tenesmus and constipation. Sigmoid cancers can mimic diverticulitis, presenting with pain, fever, and obstructive symptoms. At least $20 \%$ of patients with sigmoid cancer also have diverticular disease, making the correct diagnosis difficult at times. Sigmoid cancers can also cause colovesical or colovaginal fistulas.

In our series, most of the colorectal cancer cases presented with the feature of intestinal obstruction demanding urgent laparotomy. On the laparotomy most of the cases had massively dilated colon proximal to the constrictive growth. Few cases had bowel perforation at and proximal to the site of growth. Colonoscopy is the gold standard for establishing the diagnosis of colon cancer in elective setting. In patients with tumors causing complete obstruction, the diagnosis is most properly established by resection of the tumor without the benefit of preoperative colonoscopy. Colonoscopy permits biopsy of the tumor to verify the diagnosis while allowing inspection of the entire colon to exclude metachronous polyps or cancers. ${ }^{2}$ The aim of this study was to compare the surgical outcome and clinical profiles of emergency and elective surgical cases for colorectal cancer.

\section{METHODS}

This is a retrospective study comparing the surgical outcome and clinical profile of elective versus emergency colorectal cancer cases from December 2011 to January 2013. This study was conducted at College of medical Sciences and Teaching Hospital (COMS-TH), Bharatpur Nepal after clearance from the ethical committee of the hospital. Total of 38 cases were operated with pre or post op diagnosis of colorectal malignancy however but only 34 cases of colorectal cancer were included in the study because the data available in the record sheet for 4 cases were inadequate for the analysis and thus excluded from the study. 
Kumar $S$ et al. Surgical outcome and clinical profile of emergency

In our study, criteria for emergency case was defined as a patient who underwent emergency colorectal cancer (CRC) surgery because of perforation, obstruction, or bleeding, regardless of the time elapsed from hospital admission to operation. Remaining all the cases was considered elective. Perforation was defined as pneumoperitoneum on preoperative radiography. Obstructive tumors were defined as tumors causing intestinal obstruction. Bleeding was defined as blood loss from the tumor causing such severe anemia that the patient had to be treated by any means of surgery. The operative notes were reviewed and recorded. All the histopathological specimen reports were recorded and data entered. Pathological staging and other parameters like site of tumors and grading of tumors were obtained. Data obtained were analyzed using SPSS Statistics (version 20 for Windows; SPSS/IBM, Chicago, IL, USA). Early mortality included all deaths occurring within 30 days of surgery. According to the data available, patients were followed every 3 months after surgery and the significant findings which reflect the tumor recurrence were recorded. Outcome of both emergency and elective surgery were compared in term of post operative complications.

\section{RESULT}

The total number of patients included in this study was 34. Out of which $52.94 \%(n=18)$ were emergency cases and $47.05 \%(n=16)$ were elective. Male female ratio was 3:1in emergency cases and 2.6:1 in elective cases. Most of the populations in both the groups were between 40-60 years. Per rectal bleeding (56\%) and altered bowel habit $(31.25 \%)$ was predominant clinical presentation in elective cases whereas intestinal obstruction $(55.55 \%)$ and peritonitis $(22.22 \%)$ were predominant clinical presentation in emergency cases.

In emergency cases most of the tumors were located in left side $(77.77 \%)$ and in elective cases rectum was common site $(37.5 \%)$. Left hemicolectomy was the commonest surgery performed $(72.22 \%)$, in $22.22 \%$ cases right hemicolectomy and in $5 \%$ case diversion procedure was done in emergency set up. In elective cases, right hemicolectomy was done in $31.25 \%$ cases, the same number of the cases underwent left hemicolectomy, APR was done in $25 \%$ cases and LAR was done in $25 \%$ cases. Majority of the specimen on histopathology report was staged as stage III and IV in the emergency surgery where as it was stage I and II in majority of elective cases. This is summarized in table 3. Patient with left hemicolectomy done in emergency setup had longest duration of hospital stay of more than 18 days compared to 9 days for elective cases which was statistically significant $(\mathrm{p}<0.001)$. In the emergency group $11.11 \%$ developed enterocutaneous fistula whereas none of the elective cases developed it. Wound infection was seen in $16.66 \%$ in emergency group and $11.11 \%$ in elective group. Respiratory tract infection was seen in $6 \%$ of elective cases only. Early mortality within 30 days was observed in $5 \%$ of emergency cases only. Table

Table 1: Major predominant clinical features of colorectal cancer

\begin{tabular}{|c|c|c|c|c|c|c|c|}
\hline $\begin{array}{l}\text { Mode of } \\
\text { Clinical } \\
\text { presentation }\end{array}$ & $\begin{array}{l}\text { Abdominal } \\
\text { mass }\end{array}$ & $\begin{array}{l}\text { Altered bowel } \\
\text { Habits }\end{array}$ & $\begin{array}{l}\text { Per rectal b } \\
\text { leeding }\end{array}$ & Tenesmus & $\begin{array}{l}\text { Intestinal } \\
\text { obstruction }\end{array}$ & Peritonitis & TOTAL \\
\hline Emergency & 0 & 0 & 1 & 3 & 10 & 4 & 18 \\
\hline Elective & 2 & 5 & 9 & 0 & 0 & 0 & 16 \\
\hline TOTAL & 2 & 5 & 10 & 3 & 10 & 4 & 34 \\
\hline
\end{tabular}


Journal of College of Medical Sciences-Nepal, 2013, Vol-9, No-2,

Table 2: Type of surgery performed

Types of surgery

Right hemicolectomy

Left hemicolectomy

Low Anterior Resection (LAR)

Abdominal Peritoneal Resection (APR)

Diversion procedure(Stoma Creation)

Total
Emergency

4

13

0

0

1

18
Elective

5

5

2

4

0

16

Table 3: Major clinical variables in elective and emergency colorectal cancer

$\begin{array}{ll}\text { SN } & \text { Particulars } \\ 1 & \text { Age group(Years) }\end{array}$

2 Gender

3 Tumor Location /Site of tumor

4 Tumor Grade

4: Site of Lesions

\section{Elective}

$40-60(n=15)$

Male $(n=12)$

Female $(n=4)$

Right $(n=4)$

Left ( $n=9)$

Rectal $(\mathrm{n}=3)$

StageI $(n=2)$

SatgeII $(n=10)$

StageIII $(n=4)$

\section{Emergency}

$40-60(n=9)$

$61-80(n=8)$

Male $(n=13)$

Female $(\mathrm{n}=5)$

Right ( $\mathrm{n}=5)$

Left $(n=13)$

Stage II $(\mathrm{n}=1)$

Stage III $(n=11)$

Stage IV $(n=6$ Table

Table 4: Site of Lesions

$\begin{array}{lccr} & \text { Right } & \text { Left } & \text { Rectum } \\ \text { Elective } & 5 & 5 & 6 \\ \text { Emergency } & 4 & 14 & -\end{array}$

Table 5: Postoperative complications in elective and emergency colorectal cancer

\begin{tabular}{|c|c|c|c|c|c|c|}
\hline & \multicolumn{3}{|c|}{ Emergency } & \multicolumn{3}{|c|}{ Elective } \\
\hline & Right sided & Left sided & Rectal & Right sided & Left sided & Rectal \\
\hline $\begin{array}{l}\text { Enterocutaneous } \\
\text { fistula }\end{array}$ & 1 & 1 & - & - & - & - \\
\hline $\begin{array}{l}\text { Respiratory } \\
\text { tract infection }\end{array}$ & - & - & - & - & 1 & - \\
\hline Wound Infection & 1 & 2 & - & 1 & 1 & - \\
\hline Death & - & 1 & - & - & - & - \\
\hline
\end{tabular}

Compared with the elective patients, the emergency patients had more tumors of AJCC stages II to IV (P<0.0001 for stages III and IV) than stage I. 
Kumar S et al. Surgical outcome and clinical profile of emergency

\section{DISCUSSION}

Emergency surgery for colorectal cancer is associated with a higher risk for metastatic disease, possibly because of occult liver metastases. ${ }^{3,4}$ Although such cancers do not necessarily show a higher rate of local recurrence. ${ }^{3}$ In our study the mean age patients presenting to our hospital were 41-60 years in both the study groups which is consistent with the study conducted by Wong SK et al. in which it was found that patients undergoing emergency surgery were generally older than elective cases (mean age 68.6 and 66.3 years, respectively). In our cases male sex was predominant, which is in-accordance with the study by Wong SK et al where there was female predominance $(50.3 \%$ and $43 \%$, respectively). In one study, the 5-year overall survival rate following emergency surgery was $39.2 \%$ compared with $64.7 \%$ for elective cases. ${ }^{5}$ In our study 5 years survival could not be commented due to short duration of study.

Approximately 15 to $30 \%$ of colorectal cancers present as an emergency, most often as obstruction or perforation. Rectal cancers seldom present as an emergency (5.9\%). ${ }^{5}$ Risk for obstruction seems to be highest at the splenic flexure. ${ }^{7}$ In our study, $52.94 \%$ cases presented in emergency state. In our study the sigmoid colon was the commonest site of perforation which is consistent to with study done by Bass G et al. ${ }^{7}$ Studies report poorer outcome for patients who undergo emergency compared with elective surgery, both for their initial hospital stay and their long-term survival. In our study the noted mortality was seen in emergency case.

Emergency tumors tended to be of higher AJCC stage (II to IV), T stage (T4), and N stage (N1 to 2/
3) which is similar as shown in other literature by Wong SK et al. ${ }^{5}$ This is probably the first paper from our institution so it serves as a pilot study on emergency surgery for CRC of its kind.

\section{CONCLUSION}

In emergency conditions, colorectal cancer presented with intestinal obstruction and peritonitis where as elective cases presented with per rectal bleeding and altered bowel habits. Compared with the elective patients, the emergency patients had higher rate of morbidity and mortality and higher grade of the tumor which resulted in poor outcome. Because of higher incidence of colorectal cancer in our institute in emergency cases we have to rule out CRC in elderly patients who present with features of intestinal obstruction and peritonitis. We should develop screening programmes like Colonoscopy and biopsy to detect early colorectal cancer to improve outcome of the surgical procedures.

\section{REFERENCES}

1. Hady HR, Soldatow M, Lukaszewicz J, Luba M, Pierko J, Mysliwiec P, et al. Surgical treatment of malignant and benign colorectal neoplasms based on authors' clinical data. $A d v$ Clin Exp Med. 2013;22:219-27.

2. Robert DF,Mahmoud NN,Maron DJ,Joshua ISB. Colon and rectum In: Townsend CM, Beauchamp RD, Evers BM et al, editors. Sabiston Text Book of Surgery The Biological Basis of Modern Surgical Practice. 19th ed. Philadelphia:Elsevier Saunders.2012;12941380. 
Journal of College of Medical Sciences-Nepal, 2013, Vol-9, No-2,

3. Carraro PG, Segala M, Cesana BM, Tiberio G. Obstructing colonic cancer: failure and survival patterns over a ten-year follow-up after one-stage curative surgery. Dis Colon Rectum. 2001 ;44:243-50.

4. Biondo S, Marti-Rague J, Kreisler E, Pares D, Martin A, Navarro M, et al. A prospective study of outcomes of emergency and elective surgeries for complicated colonic cancer. Am J Surg. 2005 ;189:377-83.

5. Wong SK, Jalaludin BB, Morgan MJ, Berthelsen AS, Morgan A, Gatenby AH, et al. Tumor pathology and long-term survival in emergency colorectal cancer. Dis Colon Rectum. 2008 ;51:223-30.

6. Lee YM, Law WL, Chu KW, Poon RT. Emergency surgery for obstructing colorectal cancers: a comparison between right-sided and left-sided lesions. J Am Coll Surg. 2001;192:719-25.

7. Bass G, Fleming C, Conneely J, Martin Z, Mealy K. Emergency first presentation of colorectal cancer predicts significantly poorer outcomes: a review of 356 consecutive Irish patients. Dis Colon Rectum. 2009;52:678-84. 